\title{
Effects of a Robot-aided Somatosensory Training on Proprioception and Motor Function in Stroke Survivors
}

\section{I-Ling Yeh}

Singapore Institute of Technology https://orcid.org/0000-0001-7997-8682

Jessica Holst-Wolf

University of Minnesota School of Kinesiology

\section{Naveen Elangovan}

University of Minnesota School of Kinesiology

\section{Anna Vera Cuppone}

Istituto Italiano di Tecnologia Robotics Brain and Cognitive Science Unit

\section{Kamakshi Lakshminarayan}

University of Minnesota Department of Neurology and School of Public Health

\section{Leonardo Capello}

Scuola Superiore Sant'Anna

\section{Lorenzo Masia ( $\boldsymbol{\nabla}$ lorenzo.masia@ziti.uni-heidelberg.de)}

Institut für Technische Informatik, Universität Heidelberg, Heidelberg, Germany https://orcid.org/00000003-2402-7139

\section{Jürgen Konczak}

University of Minnesota School of Kinesiology

\section{Research}

Keywords: human, somatosensation, upper limb, rehabilitation, cerebrovascular disease/stroke

Posted Date: October 13th, 2020

DOI: https://doi.org/10.21203/rs.3.rs-89369/v1

License: (9) This work is licensed under a Creative Commons Attribution 4.0 International License. Read Full License

Version of Record: A version of this preprint was published at Journal of NeuroEngineering and Rehabilitation on May 10th, 2021. See the published version at https://doi.org/10.1186/s12984-02100871-x. 


\section{Abstract}

Background - Proprioceptive deficits after stroke are associated with poor upper limb function, slower motor recovery, and decreased self-care ability. Improving proprioception should enhance motor control in stroke survivors, but current evidence is inconclusive. Thus, this study examined whether a robot-aided somatosensory-based training requiring increasingly accurate active wrist movements improves proprioceptive acuity and motor performance in chronic stroke.

Methods - Twelve adults with chronic stroke completed a 2-day training (age range: 42 - 74 years; median time-after-stroke: 12 months; median Fugl-Meyer UE: 65). Retention was assessed at Day 5. Grasping the handle of a wrist-robotic exoskeleton, participants trained to roll a virtual ball to a target through continuous wrist adduction/abduction movements. During training vision was occluded, but participants received real-time, vibro-tactile feedback on their forearm about ball position and speed. Primary outcome was the just-noticeable-difference (JND) wrist position sense threshold as a measure of proprioceptive acuity. Secondary outcomes were spatial error in an untrained wrist tracing task and somatosensory-evoked potentials (SEP) as a neural correlate of proprioceptive function. Ten neurologically-intact adults were recruited to serve as non-stroke controls for age, gender and hand dominance (age range: 44 to 79 years; 6 women, 4 men).

Results - Participants significantly reduced JND thresholds at posttest and retention $(\mathrm{F}(2,38)=4.54, p=$ $0.017, \eta_{p}{ }^{2}=0.20$ ) in both groups. A higher pretest JND threshold was associated with a higher threshold reduction at posttest and retention $(r=-0.86,-0.90, p \leq 0.001)$ among the stroke participants. Error in the untrained tracing task was reduced by $22 \%$ at posttest, yielding an effect size of $w=0.13$. Stroke participants exhibited significantly reduced P27-N30 peak-to-peak SEP amplitude at pretest $(U=11, p=$ 0.03) compared to the non-stroke group. SEP measures did not change systematically with training.

Conclusion - This study provides proof-of-concept that non-visual, proprioceptive training can induce fast, measurable improvements in proprioceptive function in chronic stroke survivors. There is encouraging but inconclusive evidence that such somatosensory learning transfers to untrained motor tasks.

Trial Registration Clinicaltrials.gov; Registration ID: NCT02565407; Date of registration: 01/10/2015; URL: https://clinicaltrials.gov/ct2/show/NCT02565407

\section{Background}

Nearly two-thirds of stroke survivors exhibit forms of somatosensory or proprioceptive dysfunction $(1,2)$. Proprioceptive deficits are related to longer length-of-stay in hospitals(3), more severe upper limb impairment, and decreased activities of daily living (ADL) (4-6). Given the crucial role of proprioception for motor control, restoring proprioceptive function should enhance motor recovery after stroke. Proprioceptive training is a form of somatosensory intervention that aims to enhance proprioceptive function (7). Several forms of somatosensory intervention such as passive cutaneous electrical stimulation, passive limb movement training, repeated somatosensory discrimination practice and active 
sensorimotor training with augmented somatosensory feedback (8) have been proposed to aid recovery of proprioceptive-motor function after stroke (7). Improvements observed in proprioceptive function after proprioceptive training interventions correlated with improved untrained motor performance in healthy adults (9) further supporting the rationale to implement proprioceptive-motor training in stroke.

Within the framework of a somatosensory-based motor training, somatosensory signals are employed to provide movement feedback for online motor control either in the absence of vision or to augment existing visual feedback. Using augmented somatosensory feedback during voluntary movement will activate a well-known network of somatosensory cortical and motor cortical areas $(10,11)$. One specific type of somatosensory feedback is vibro-tactile feedback (VTF) applied superficially to the skin. Previous studies on healthy adults documented that VTF can effectively enhance wrist proprioceptive function and can improve motor learning outcomes $(12,13)$. To understand the physiology of sensorimotor changes following proprioceptive training, it is desirable to obtain neural markers of somatosensory processing reflecting the change in neural response to such somatosensory-based intervention. Somatosensory evoked potentials (SEPs) collected via electroencephalography (EEG) constitute a simple, established neural marker of somatosensory processing to explore the underlying neural responses following proprioceptive training in adults with stroke.

To address if a somatosensory-based training is a meaningful approach for the rehabilitation of proprioceptive and motor function after stroke, this proof-of-concept study aimed to determine whether wrist proprioception could be trained in stroke survivors and whether such sensory learning transfers to other functional tasks involving the same joint and limb motor system.

\section{Methods}

\section{Participants}

Twelve stroke survivors at least three months post cerebral stroke were recruited (Table 1). Study inclusion required an ability to achieve $20^{\circ}$ wrist ab/adduction at the more-affected side with minimal resistance in the gravity-eliminated position (score of $2+/ 5$ in manual muscle testing) (14). Exclusion criteria were: 1) non-cerebral stroke, 2) <23 points on Mini Mental State Examination (15), 3) markedly increased muscle tone as indicated by $>1+$ on the Modified Ashworth Scale (16), 4) other medical conditions affecting upper limb sensorimotor function, 5) inability to perceive VTF on either forearm, and 6) lack of MRI records confirming stroke diagnosis. Participants were recruited via an outpatient neurology clinic, local stroke support groups, and mailing to addresses retrieved from the clinical data depository of the University of Minnesota Clinical and Translational Science Institute (Figure 1). All participants lived at home in independent self-care. Ten neurologically-intact adults matched by age, gender, and hand dominance were recruited to serve as non-stroke controls for age and gender (Median age: 71 years, range: 44 to 79 years; 6 women, 4 men). The study protocol was approved by the Human Research Protection Program of University of Minnesota. Written informed consent was obtained from all 
participants prior to data collection. Study data are available from the corresponding author upon reasonable request. 
Table 1

Demographics and clinical evaluation for participants with stroke

\begin{tabular}{|c|c|c|c|c|c|c|c|}
\hline ID & Gender & $\begin{array}{l}\text { Age } \\
\text { (years) }\end{array}$ & $\begin{array}{l}\text { Time post } \\
\text { stroke } \\
\text { (months) }\end{array}$ & $\begin{array}{l}\text { Lesion } \\
\text { side }\end{array}$ & Lesion location & Type & $\begin{array}{l}\text { FMA- } \\
\text { UL } \\
(0- \\
66)\end{array}$ \\
\hline S03 & W & 57 & 27 & L & $\begin{array}{l}\text { Cortical and } \\
\text { subcortical parietal } \\
\text { lobe }\end{array}$ & ischemic & 66 \\
\hline S04 & M & 73 & 11 & L & EC, putamen, PVWM & ischemic & 66 \\
\hline S05 & M & 47 & 4 & L & $\begin{array}{l}\text { Posterior subcortical } \\
\text { frontal, BG, posterior } \\
\text { limb of IC }\end{array}$ & ischemic & 65 \\
\hline S06 & W & 74 & 6 & $\mathrm{R}$ & Thalamus, putamen & hemorrhagic & 64 \\
\hline S07 & $\mathrm{M}$ & 63 & 7 & L & Corona radiate & ischemic & 65 \\
\hline S08 & W & 42 & 13 & $\mathrm{R}$ & $\begin{array}{l}\text { Superior thalamus, } \\
\text { cortical and } \\
\text { subcortical temporal } \\
\text { and occipital lobe }\end{array}$ & ischemic & 64 \\
\hline S09 & W & 63 & 5 & $\mathrm{R}$ & $\begin{array}{l}\text { Frontal (precentral } \\
\text { gyrus), parietal } \\
\text { (postcentral gyrus), } \\
\text { occipital lobe }\end{array}$ & ischemic & 66 \\
\hline S10 & M & 65 & 26 & $L \& R$ & $\begin{array}{l}\text { Cortical and } \\
\text { subcortical occipital } \\
\text { lobe, L \& R thalamus }\end{array}$ & ischemic & $46^{*}$ \\
\hline S11 & M & 71 & 55 & $\mathrm{R}$ & Thalamus & hemorrhagic & $42^{\star}$ \\
\hline S12 & W & 68 & 6 & L & $\begin{array}{l}\text { Frontal (precentral } \\
\text { gyrus) }\end{array}$ & ischemic & 65 \\
\hline S13 & $\mathrm{M}$ & 60 & 49 & L & $\begin{array}{l}\text { Subcortical frontal } \\
\text { and parietal }\end{array}$ & ischemic & 58 \\
\hline S14 & W & 56 & 14 & L & $\begin{array}{l}\text { Frontal (precentral } \\
\text { gyrus), parietal } \\
\text { (postcentral gyrus) }\end{array}$ & ischemic & 64 \\
\hline Ave. & $\begin{array}{l}6 \\
\text { Women/6 } \\
\text { Men }\end{array}$ & 62 & 18 & $\begin{array}{l}4 \mathrm{R} / 7 \\
\mathrm{~L} / 1 \\
\text { both }\end{array}$ & $\begin{array}{l}3 \text { cortical / } 7 \\
\text { subcortical/ } 2 \text { both }\end{array}$ & 10 ischemic & 61 \\
\hline $\begin{array}{l}\text { Note. } \\
\text { Asse } \\
\text { periv }\end{array}$ & $\begin{array}{l}\text { npaired wr } \\
\text { nent. FMA } \\
\text { ricular whi }\end{array}$ & positio & $\begin{array}{l}\text { sense indica } \\
\text { er Assessm } \\
\text {, basal gan }\end{array}$ & $\begin{array}{l}\text { ed by Ero } \\
\text { ent Uppe }\end{array}$ & $\begin{array}{l}\text { nus MC modified Nott } \\
\text { imb. EC, external caps } \\
\text { nal capsule. }\end{array}$ & $\begin{array}{l}\text { ham Sensory } \\
\text { PVWM, }\end{array}$ & \\
\hline
\end{tabular}




\section{Study Design}

The study employed a pre-post design with a single control group. Participants completed the pretest and one intervention session on Day 1, the second intervention and the posttest on Day 2 with retention testing at Day 5 (Figure 2A).

\section{Apparatus}

A wrist/hand exoskeleton robotic system (the WristBot) allowing full ROM at three degrees-of-freedom (DOF; wrist flexion/extension, wrist abduction/adduction and forearm pronation/supination; see Figure 2B) was used for training and assessment (for a full technical description of the robot see $(17,18)$ ). It generated appropriate torques to passively displace the hand smoothly to a joint position. Optical encoders measured angular displacement at a high resolution $\left(0.0065^{\circ}\right.$ in wrist abduction/adduction (AA); $0.0075^{\circ}$ in wrist flexion/extension (FE) (19). Robotic control was implemented through Matlab Simulink code (MathWorks, Natick, Massachusetts, USA).

\section{Intervention}

Participants sat on a height-adjustable chair. The medial-lateral wrist joint axis was aligned with the axis of rotation of the WristBot. During intervention, only one degree of freedom - AA was trained. Participants grasped the robot handle and made continuous, small amplitude wrist AA movements to position a virtual ball into a target area on a tilt-able table viewed on a display (Figure 2B) the rotation scaling factor was set to translate each degree of wrist motion to one degree of tilt angle of the virtual table. Motion of the virtual ball towards the target was generated by tilting the table using FE and AA corresponding to the two coordinated axes of rotations: the dynamics of the virtual ball was simulated by considering its mass and gravity force generated by the inclination of the table and, consequently imposing a kinematics on its trajectory. Participants completed a single 24-minute session on Day1 and Day2 for a total of 48 minutes of training. Both sessions began with a 5-minute familiarization phase, then continued with three 8minute training blocks. During the familiarization phase, participants learned to associate VTF with the ball-target distance and ball speed in the presence of visual feedback of the virtual table/ball system. After familiarization with vision, participants continued practicing with eyes closed for the remainder of training relying solely on vibro-tactile feedback to complete the task of moving the virtual ball to the target zone. VTF was provided by three light-weight vibratory motors $(9 \mathrm{~mm}$ in diameter, $25 \mathrm{~mm}$ in length, 4.6 g; Model 307-100, Precision Microdrives Ltd., London, United Kingdom). Two vibratory motors were placed along the longitudinal axis of the training forearm at a distance that users verbally reported that they could differentiate (Figure 2B). They encoded ball position and distance of the ball relative to the target. Vibration frequency increased in three levels $(80,90$, trains of $100 \mathrm{~Hz}$ pulses) with the distance from the ball to the target. Preliminary work from our group established that a $10 \mathrm{~Hz}$ difference in signal is discernible on the forearm and all participants reported that they were able to differentiate the differences 
in vibration frequency. The distal vibrator turned on when the ball was on the right side of the target, while the proximal vibration motor was switched on when the ball was the left side of target. A third vibrator placed on the non-performing forearm encoded ball speed by vibrating between 75-98 Hz (Higher frequency indicated higher ball speed). A trial was completed when the ball stayed within the target area for 5 seconds. Target locations on the table changed between trials (left, center, right). The difficulty level increased after every 6 trials by altering the virtual dynamics of the system (i.e., increasing ball mass, decreasing table friction).

\section{Primary Outcome Measure}

\section{Just-noticeable difference (JND) threshold}

Participants sat in a height-adjustable chair. The tested forearm was secured with a Velcro strap to the splint of the robot to minimize movement during testing. Vision was occluded. Pink noise provided via headphones masked external sounds that could provide position information. The participant's wrist was displaced from an initial position of $10^{\circ}$ wrist adduction (ulnar deviation) at a constant angular velocity of $6^{\circ} \mathrm{s}$. Two stimuli were presented in each trial: A reference position of $5^{\circ}$ wrist abduction and a comparison position. The comparison position was always more abducted than the reference position. The order of the two positions was randomized. In each trial, participants verbally identified the stimulus with the larger amplitude in response to "Which position was the farthest from the starting position?" The stimuli difference in the subsequent trial was determined based on the participant's response by an adaptive psi-marginal algorithm (20). A correct response was followed by a smaller stimulus difference than the previous trial and vice versa. For the first trial, stimulus difference was set at $1.9^{\circ}$, which was $25 \%$ higher than the threshold of a healthy young adult cohort (19). Breaks were scheduled every 10 to 15 trials. The JND threshold represented the smallest stimulus intensity that the participant can discriminate based on the fitted performance function after 30 trials. The method's test-retest reliability was $r=0.99$. The average within-subject variability was $0.09^{\circ}(19)$.

\section{Secondary Outcome Measures}

\section{Accuracy of wrist tracing}

To examine the transfer effect of the proposed wrist proprioceptive training on the untrained motor task, participants held the handle of the device and actively traced templates presented on a computer monitor by using the WristBot to control a cursor on the monitor. Wrist flexion/extension was mapped to linear horizontal, wrist abduction/adduction on to linear vertical cursor movement. The task consisted of 5 shapes: horizontal line, vertical line, triangle, figure of eight, and ellipse (Figure 2C). Shapes were scaled to $60 \%$ of the participant's active ROM in the respective DOF to avoid confounding by end-range muscle 
tightness. The reference trace template was always visible. A target circle was presented to indicate the desired tracing direction. Participants started tracing with a wrist flexion movement. The obtained angular position time-series data were filtered offline using a low-pass $4^{\text {th }}$-order Butterworth filter (cut-off frequency: $2.5 \mathrm{~Hz}$ ). The minimal distance of each cursor sampling point with respect to the template indicated the instantaneous tracing error. For each shape, the mean and standard deviation of the instantaneous tracing errors were calculated for each participant and used as variables for subsequent analysis.

\section{Somatosensory evoked potentials}

To obtain a neural correlate of proprioceptive function, we recorded somatosensory evoked potentials. SEPs were induced by median nerve stimulation applied to the trained wrist via electrical stimulation (S88 stimulator with SIU 5 stimulus isolation unit; Grass Technologies, West Warwick, RI, USA). Square-wave pulses of $0.2 \mathrm{~ms}$ duration were delivered at $2 \mathrm{~Hz}$ and the voltage sufficient to induce a noticeable thumb adductor twitch. 1200 stimuli were delivered in two blocks, with a break at the $600^{\text {th }}$ stimulus.

EEG data were recorded continuously from nine $\mathrm{Ag} / \mathrm{AgCl}$ electrodes mounted on an elastic cap using the ANT Neuro eego system (Medical Imaging Solutions $\mathrm{GmbH}$, Berlin, Germany). The montage covered the primary sensorimotor cortical area (Fz, F3/4, FC1/2, FC3/4, Cz, C3/4, CP3/4, CP5/6, P3/4) on the contralateral hemisphere of the stimulated wrist and bilateral mastoid processes based on the standard international 10-20 system. Signals recorded from bilateral mastoid processes were used to re-reference the scalp recording offline. All signals were sampled at $2 \mathrm{kHz}$ or $4 \mathrm{kHz}$ with a 24-bit $A / D$-converter. EEG data were processed using EEGLAB (21) and ERPLAB toolboxes (22). First, continuous EEG signals were visually inspected to remove visible electromyographic or movement artifacts. Second, data were resampled to $1000 \mathrm{~Hz}$ and baseline correction was performed using the average value. Third, data were filtered using a $2^{\text {nd }}$-order Butterworth high-pass (cut-off: $0.1 \mathrm{~Hz}$ ) and a low-pass (cut-off: $200 \mathrm{~Hz}$ ) filter in series. Signals were then re-referenced to the average signals recorded from bilateral mastoid processes. Lastly, the continuous signals were segmented into 300-ms epochs with $100 \mathrm{~ms}$ before and $200 \mathrm{~ms}$ after the onset of the electrical stimulus. Artifact rejection was performed through a moving average method that flagged epochs containing peak-to-peak amplitudes higher than $100 \mu \mathrm{V}$ in 200-ms moving window in a 100-ms step. Artifact-free epochs were then averaged to generate the grand average for each participant session (89\% of total epochs were accepted after artifact rejection).

Three measures of early somatosensory cortical processing were extracted based on the individual SEP waveforms for each participant: (1) peak latency of N30, defined as the first negative peak from the frontal electrodes (F3/4, FC1/2, FC3/4) after 28 ms (23), (2) peak-to-peak amplitude of P27-N30, and (3) P45, where P27 refers to the positive peak prior to N30, occurring between 22-28 ms after stimulus (24). P45 is the positive peak following N30.

\section{Statistical Analysis}


Distributions of JND across the three measurements were not significantly different from normal distribution based on Shapiro-Wilk tests ( $p$ values $>0.05$ ) and a $2 \times 3$ (group: stroke and control $x$ measurement time [pretest, posttest and retention]) mixed ANOVA was performed to examine the change of JND over time with the comparison between the stroke and control groups. Pearson correlation coefficients $(r)$ were computed for bivariate analysis of JND thresholds. For other variables, half of the tracing errors were not normally distributed as indicated by Shapiro-Wilk test. Even with one outlier $(>2$ interquartile range [IQR]) removed across the tasks, distributions of six variables were still not normally distributed. The SEP variables N30 peak-to-peak amplitude, N30 latency, and P45 latency were not normally distributed as indicated by Shapiro-Wilk test. Therefore, to account for non-normal distribution, nonparametric Friedman tests were employed to examine changes in tracing errors and all SEP variables over the three measurements for the stroke and control groups respectively. Kendall's $w$ was calculated to indicate the effect size of Friedman's test by normalizing the chi square statistics obtained in the Friedman's test by the number of participants $(\mathrm{N})$ and degrees of freedom (i.e. the number of repeated measures -1$)(25)$. Kendall's $w$ indicates the percentage of variance among the ranks explained by the repeated measures, similar to eta squared used in ANOVA designs. Spearman correlation coefficients $\left(r_{s}\right)$ were computed for bivariate analysis for tracing errors and SEP measures. Significance level was set at a $=0.05$.

\section{Results}

All participants successfully completed both the training and assessment sessions with no discomfort. S10 could not perceive passive wrist movement of the affect side without vision and therefore could not complete JND test. However with VTF placed on the less-affected forearm, S10 could complete the training.

\section{JND Threshold as a Marker of Wrist Proprioceptive Acuity}

Eleven of the twelve stroke participants completed the threshold testing across the three visits. S10 did not complete the JND test because he could not perceive passive wrist movement without vision on the affected side. At pretest, mean JND was $1.8^{\circ}$ [SD: $\left.0.54^{\circ}\right]$ for the stroke and $1.5^{\circ}\left[0.46^{\circ}\right]$ for the control group with seven stroke participants exhibiting a JND threshold higher than the median JND threshold of the control group (see Figure 3A). At posttest, 8 out of 11 stroke participants (73\%) lowering their thresholds (see Figure 3), which reduced the JND threshold at group level (mean: $1.4^{\circ}$ [SD: $\left.0.54^{\circ}\right]$ ). The 8 participants, who responded to somatosensory training, reduced their JND threshold between 9.3-47.0\% (mean: $30.2 \%$ ). Two participants showed a slight increase (2.5-6.6\%) in threshold, while one participant increased his threshold by $48 \%$. At retention, the reduction in JND threshold remained stable (mean: $1.3^{\circ}$ [SD: $\left.0.28^{\circ}\right]$ for the stroke group), indicating that improvements in JND threshold persisted two days after the intervention. Mauchly's Test of Sphericity indicated that the assumption of sphericity had been not violated $\left(X^{2}(2)=1.40, p=0.497\right)$ and therefore no correction was used for $2 \times 3$ mixed ANOVA on JND. Aligned with the above observation, the analysis revealed a significant main effect of measurement time 
$\left(\mathrm{F}(2,38)=4.54, p=0.017, \eta_{\mathrm{p}}^{2}=0.19\right)$ while indicating no group and time interaction $(\mathrm{F}(2,38)=1.74, p=$ $\left.0.189, \eta_{p}{ }^{2}=0.08\right)$ and no main effect of group $\left(F(1,19)=0.12, p=0.736, \eta_{p}^{2}=0.06\right)$. It suggested significant reduction in JND across measurement time for both groups. Trend analysis suggested a significant linear trend for the changes across measurement time $\left(F(1,19)=6.60, p=0.019, \eta_{p}^{2}=0.26\right)$. A high pretest JND threshold was the strongest predictor of improvement in JND at posttest $(r=-0.71, p=$ $0.015)$ and at retention $(r=-0.80, p=0.003$; Figure $3 \mathrm{~B})$.

\section{Wrist Tracing Accuracy as a Marker of Untrained Visuomotor Performance}

At pretest, the stroke group exhibited significantly higher tracing errors when tracing the triangle $(U=17, p$ $=0.005)$, figure-of-eight $(U=22, p=0.012)$ and ellipse $(U=22, p=0.012)$ compared to the control group. At posttest, participants of the stroke group showed $22 \%$ reduction in tracing error on average compared to pretest. However the observed reduction did not achieve statistical significance as indicated by Friedman's tests $\left(\chi^{2}(2)=0.30-3.17, p\right.$ values $=0.205-0.407$ for the stroke group; $\chi^{2}(2)=1.80-5.00, p$ values $=0.082-0.407$ for the control group). Effect sizes indicated by Kendall's $w$ ranged from 0.01 to 0.13 for the stroke group and 0.09 - 0.25 for the non-stroke group, indicating low to medium effects. Vertical line and triangle tracing exhibited the highest effect size in the stroke group (Figure 4B). Pretest tracing error of the ellipse correlated significantly with JND threshold at pretest $\left(r_{s}=-0.62, p=0.043\right)$ among the stroke group, indicating association between proprioceptive acuity and motor tracing performance.

\section{SEP as a Marker of Somatosensory Processing}

SEP waveforms were obtained from FC1 for optimal observation of the N30 waveform for the nine stroke participants and seven control participants who completed the SEP test. The remaining participants declined due to time pressure and fatigue as SEP was conducted last. Participants with stroke showed a significantly reduced P27-N30 peak-to-peak amplitude (median: $2.07 \mu \mathrm{V}$ [IQR: $2.94 \mu \mathrm{V}$ ]) when compared to controls $(4.47 \mu \mathrm{V}[3.84 \mu \mathrm{V}], U=11, p=0.03$; Figure 5A, 5C). After training, median P27-N30 peak-topeak amplitude for the stroke group increased from $2.07 \mu \mathrm{V}(2.94 \mu \mathrm{V})$ at pretest to $2.29 \mu \mathrm{V}(1.92 \mu \mathrm{V})$ at posttest and to $2.87 \mu \mathrm{V}(1.73 \mu \mathrm{V})$ at retention (40\% increase) but this increase failed to reach statistical significance at group level $\left(\chi^{2}(2)=2.00, p=0.37, w=0.11\right)$. In contrast of the significant group difference of P27-N30 peak-to-peak amplitude, N30 latency of six in nine stroke participants were within the range of the control group. After training, median N30 latency of both groups did not change at posttest (stroke: 39 $\mathrm{ms}$ [10 ms]; control: $32 \mathrm{~ms}$ [7 ms]) and retention (stroke: $39 \mathrm{~ms}$ [12 ms]; control: $32 \mathrm{~ms}$ [5 ms]) compared to pretest (stroke: $39 \mathrm{~ms}$ [9.5 ms]; control: $32 \mathrm{~ms}$ [7 ms]). This was confirmed by Friedman's tests (stroke: $\chi^{2}(2)=0.58, p=0.75, w=0.03$; control: $\chi^{2}(2)=1.08, p=0.58, w=0.08$ ). Further subgroup analysis found that at pretest four of the five stroke participants with more affected proprioceptive acuity $(\mathrm{S} 05, \mathrm{~S} 06, \mathrm{~S} 08$, 
S14; JND threshold $>=1.8^{\circ}$ ) showed delayed latency of either N30 or P45 component. In this subgroup, a longer P45 peak latency was correlated with a higher JND threshold at pretest $\left(r_{s}=0.66, p<0.05\right.$; Figure $5 B$ for example).

\section{Discussion}

This proof-of-concept study sought to provide initial data on the assumed effectiveness of somatosensory-focused, active movement interventions for improving sensorimotor function in chronic stroke. The main findings of the study are as follows: First, a short, 2-day movement training that challenges the proprioceptive system lead to measurable improvements in wrist position sense accuracy that persisted for at least 3 days past training. Second, evidence for a transfer of such sensory learning to the motor domain is limited. Stroke participants exhibited a $22 \%$ mean reduction in tracing error, which is promising. However, given the high response variability of the stroke group, this difference was not statistically significant. Third, a reduced SEP amplitude during early somatosensory processing is a marker of abnormal proprioceptive processing. However, the short training did not induce a systematic change in SEP amplitude as a sign of normalized cortical processing.

\section{A somatosensory-focused movement training can improve proprioceptive acuity in stroke survivors}

We found that $73 \%(8 / 11)$ of stroke participants responded to the two-day somatosensory-based training. For those responders, wrist proprioceptive acuity improved by $30 \%$ on average. Importantly, these gains in sensory acuity persisted for two days. This finding is encouraging as it demonstrates that a brief somatosensory-focused training can induce measurable benefits in proprioceptive function in chronic phase stroke survivors.

Our data provide initial evidence that for adults with chronic stroke an active, somatosensory-focused movement training may require less training time to achieve comparable proprioceptive improvement than either somatosensory discrimination training alone or functional arm use exercises combined somatosensory discrimination training. Research for those types of training reported that a reduction of $21 \%-67 \%$ in joint reposition error took between $8-40$ hours of intervention (26-29). In comparison, our somatosensory-based movement intervention yielded a comparable magnitude of proprioceptive improvement after a total of 48 minutes of practice time.

It is important to note that the observed proprioceptive improvement is unlikely a mere practice effect of the assessment task. While the intervention required active wrist movement, the joint position discrimination task to assess proprioceptive function is based on passive motion induced by the robot. More importantly, it is inherent to psychophysical threshold testing that the perceiver receives no feedback. That is, after two distinct joint positions were assumed during our procedure, no feedback was 
given about the correctness of the participant's perceptual judgement. Thus, participants could not learn, and consequently the gains in proprioceptive acuity cannot be understood as a practice effect.

\section{Evidence of a motor transfer}

We employed a set of motor tasks that were not part of the practice regimen but used the same joint to understand if such somatosensory-focused training would transfer to untrained motor patterns. Stroke participants as a group showed on average a $22 \%$ reduction in the tracing error when compared to the pretest. However, despite a medium level of effect size this difference was not statistically significant at a group level. There are likely two reasons that account for the absence of a significant intervention effect. First, given the large between-subject movement variability generally observed in stroke survivors, the sample size of our study was insufficient. Second, the duration of the intervention was simply too short to induce systematic improvements in untrained motor tasks. Research that employed somatosensory discrimination training combined with active somatosensory-relevant exploration activities (30) reported that $18-27$ hours of intervention were necessary to yield an approximately $50 \%$ improvement in functional arm use in chronic stroke survivors with somatosensory impairment. That is, the research applying a somatosensory-focused training in a longer, more intensive regimen is necessary to generate conclusive evidence that such training can transfer to untrained functional motor tasks.

\section{SEPs reflect proprioceptive acuity at pretest}

We recorded median nerve stimulation induced SEPs to obtain a neurophysiological signal related to cortical proprioceptive processing. We investigated if electrocortical measures derived from these SEP signals were abnormal in the stroke group and if they correspond to the psychophysical position sense thresholds that measured wrist proprioceptive acuity. Specifically, we looked at markers of early somatosensory processing. Particularly, N30 is believed to be generated in the secondary somatosensory cortex (31) and evoked by the proprioceptive input (32). We found that the stroke group showed a significantly reduced P27-N30 peak-to-peak amplitude prior to training. In addition, in those stroke participants who were most affected proprioceptively, the P45 latency tended to be delayed - at pretest P45 peak latency strongly correlated with JND threshold. However, we found inconclusive evidence that these SEP components of early somatosensory processing were altered systematically at the end of our somatosensory-focused movement training.

\section{Conclusion}

This study documented that proprioceptive function is trainable and can improve in chronic stroke survivors, while the effects on motor function are encouraging but inconclusive. If proven effective in future clinical trials, such intervention or its elements could be employed in clinical practice to complement existing approaches. 


\section{List Of Abbreviations}

$A D L$, activities of daily living;

VTF, vibro-tactile feedback;

$\mathrm{AA}$, abduction/adduction;

$\mathrm{FE}$, flexion/extension;

JND, just-noticeable difference;

SEP, somatosensory-evoked potentials;

ANOVA, analysis of variance;

$\mathrm{IQR}$, interquartile range

\section{Declarations}

\section{Ethics approval and consent to participate:}

Ethics approval was obtained from the IRB of University of Minnesota. Consent to participate was obtained from each participant prior to data collection.

\section{Consent for publication:}

not applicable

\section{Availability of data and materials:}

The datasets used and/or analyzed during the current study are available from the corresponding author on reasonable request.

\section{Competing interests:}

The authors declare that they have no competing interests

\section{Funding:}

This study was supported by NIH Clinical and Translational Science award (UL1TR000114) and by a NIH Research Evaluation and Commercialization Hub award (5U01HL127479-03) to JK. Development of the 
software for the wrist robotic system was supported by Fondazione Istituto Italiano di Tecnologia.

\section{Authors' contributions:}

AVC, LC, LM; Medical screening of all participants: KL; Data collection and processing: IY; Figures and statistical analysis: IY, NE, JH; Manuscript writing and editing: IY, NE, JK.

\section{Acknowledgements:}

We sincerely thank all participants for devoting their time to this research study. The robotic device was provided by the Istituto Italiano di Tecnologia, Department of Robotics, Brain and Cognitive Sciences. Software development and support:

\section{References}

1. Kessner SS, Bingel U, Thomalla G. Somatosensory deficits after stroke: a scoping review. Top Stroke Rehabil. 2016;23(2):136-46.

2. Connell L, Lincoln N, Radford K. Somatosensory impairment after stroke: frequency of different deficits and their recovery. Clinical Rehabilitation. 2008;22(8):758-67.

3. Sommerfeld DK, von Arbin MH. The impact of somatosensory function on activity performance and length of hospital stay in geriatric patients with stroke. Clin Rehabil. 2004;18(2):149-55.

4. Tyson SF, Hanley M, Chillala J, Selley AB, Tallis RC. Sensory loss in hospital-admitted people with stroke: characteristics, associated factors, and relationship with function. Neurorehabil Neural Repair. 2008;22(2):166-72.

5. Coupar F, Pollock A, Rowe P, Weir C, Langhorne P. Predictors of upper limb recovery after stroke: a systematic review and meta-analysis. Clin Rehabil. 2012;26(4):291-313.

6. Meyer S, Karttunen AH, Thijs V, Feys H, Verheyden G. How do somatosensory deficits in the arm and hand relate to upper limb impairment, activity, and participation problems after stroke? A systematic review. Phys Ther. 2014;94(9):1220-31.

7. Aman JE, Elangovan N, Yeh IL, Konczak J. The effectiveness of proprioceptive training for improving motor function: a systematic review. Front Hum Neurosci. 2014;8:1075.

8. De Santis D, Zenzeri J, Casadio M, Masia L, Riva A, Morasso P, et al. Robot-assisted training of the kinesthetic sense: enhancing proprioception after stroke. Front Hum Neurosci. 2014;8:1037.

9. Vahdat S, Darainy M, Ostry DJ. Structure of plasticity in human sensory and motor networks due to perceptual learning. Journal of Neuroscience. 2014;34(7):2451-63.

10. Marini F, Zenzeri J, Pippo V, Morasso P, Campus C. Neural correlates of proprioceptive upper limb position matching. Human brain mapping. 2019;40(16):4813-26. 
11. Wenderoth N, Toni I, Bedeleem S, Debaere F, Swinnen SP. Information processing in human parietofrontal circuits during goal-directed bimanual movements. Neurolmage. 2006;31(1):264-78.

12. Cuppone AV, Squeri V, Semprini M, Masia L, Konczak J. Robot-Assisted Proprioceptive Training with Added Vibro-Tactile Feedback Enhances Somatosensory and Motor Performance. PLoS One. 2016;11(10):e0164511.

13. Cuppone AV, Semprini M, Konczak J. Consolidation of human somatosensory memory during motor learning. Behav Brain Res. 2018;347:184-92.

14. Hislop H, Avers D, Brown M. Daniels and Worthingham's Muscle Testing-E-Book: Techniques of Manual Examination and Performance Testing: Elsevier Health Sciences; 2013.

15. Folstein MF, Folstein SE, McHugh PR. Mini-Mental State: a practical method for grading the cognitive state of patients for the clinician: Pergamon Press; 1975.

16. Bohannon RW, Smith MB. Interrater reliability of a modified Ashworth scale of muscle spasticity. Phys Ther. 1987;67(2):206-7.

17. Masia L, Casadio M, Giannoni P, Sandini G, Morasso P. Performance adaptive training control strategy for recovering wrist movements in stroke patients: a preliminary, feasibility study. $J$ Neuroeng Rehabil. 2009;6:44.

18. Masia L, Casadio M, Sandini G, Morasso P. Eye-hand coordination during dynamic visuomotor rotations. PLoS One. 2009;4(9):e7004.

19. Cappello L, Elangovan N, Contu S, Khosravani S, Konczak J, Masia L. Robot-aided assessment of wrist proprioception. Front Hum Neurosci. 2015;9:198.

20. Prins N. The psi-marginal adaptive method: How to give nuisance parameters the attention they deserve (no more, no less). J Vis. 2013;13(7):3.

21. Delorme A, Makeig S. EEGLAB: an open source toolbox for analysis of single-trial EEG dynamics including independent component analysis. J Neurosci Methods. 2004;134(1):9-21.

22. Lopez-Calderon J, Luck SJ. ERPLAB: an open-source toolbox for the analysis of event-related potentials. Front Hum Neurosci. 2014;8:213.

23. Cruccu G, Aminoff M, Curio G, Guerit J, Kakigi R, Mauguiere F, et al. Recommendations for the clinical use of somatosensory-evoked potentials. Clinical Neurophysiology. 2008;119(8):1705-19.

24. Longo MR, Pernigo S, Haggard P. Vision of the body modulates processing in primary somatosensory cortex. Neurosci Lett. 2011;489(3):159-63.

25. Tomczak M, Tomczak E. The need to report effect size estimates revisited. An overview of some recommended measures of effect size. 2014.

26. Carey LM, Matyas TA, Oke LE. Sensory loss in stroke patients: effective training of tactile and proprioceptive discrimination. Arch Phys Med Rehabil. 1993;74(6):602-11.

27. Carey LM, Matyas TA. Training of somatosensory discrimination after stroke: facilitation of stimulus generalization. Am J Phys Med Rehabil. 2005;84(6):428-42. 
28. Byl N, Roderick J, Mohamed O, Hanny M, Kotler J, Smith A, et al. Effectiveness of sensory and motor rehabilitation of the upper limb following the principles of neuroplasticity: patients stable poststroke. Neurorehabil Neural Repair. 2003;17(3):176-91.

29. Borstad AL, Bird T, Choi S, Goodman L, Schmalbrock P, Nichols-Larsen DS. Sensorimotor training and neural reorganization after stroke: a case series. J Neurol Phys Ther. 2013;37(1):27-36.

30. Turville M, Carey LM, Matyas TA, Blennerhassett J. Change in Functional Arm Use Is Associated With Somatosensory Skills After Sensory Retraining Poststroke. American Journal of Occupational Therapy. 2017;71(3):7103190070p1-p9.

31. Barba C, Frot M, Mauguiere F. Early secondary somatosensory area (SII) SEPs. Data from intracerebral recordings in humans. Clin Neurophysiol. 2002;113(11):1778-86.

32. Restuccia D, Valeriani M, Insola A, Lo Monaco M, Grassi E, Barba C, et al. Modality-related scalp responses after electrical stimulation of cutaneous and muscular upper limb afferents in humans. Muscle Nerve. 2002;26(1):44-54.

\section{Figures}

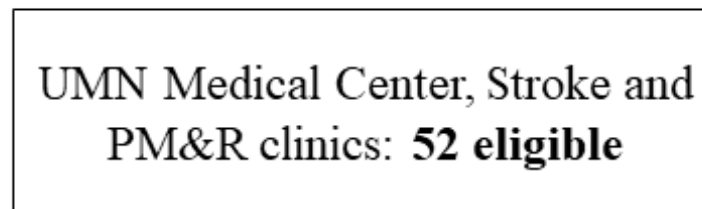

Stroke support groups, UMN StudyFinder, ClinicalTrials.gov: 20 self-referred

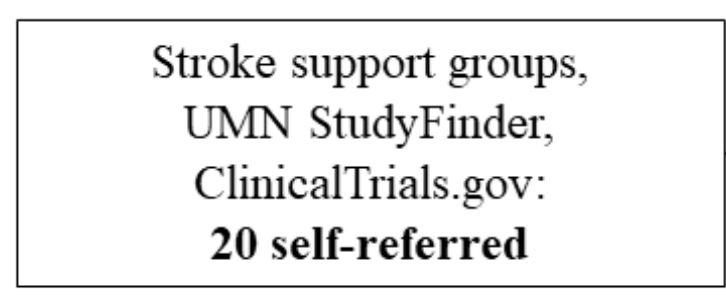

\section{Mass mailing to Clinical Data Repository, UMN/Fairview visits:} 182 eligible

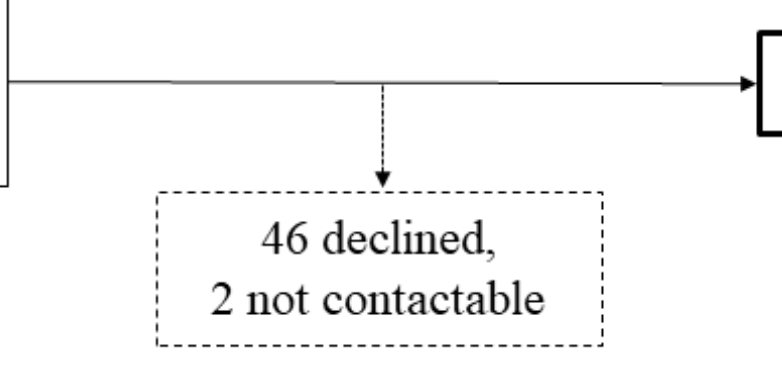

Six recruited

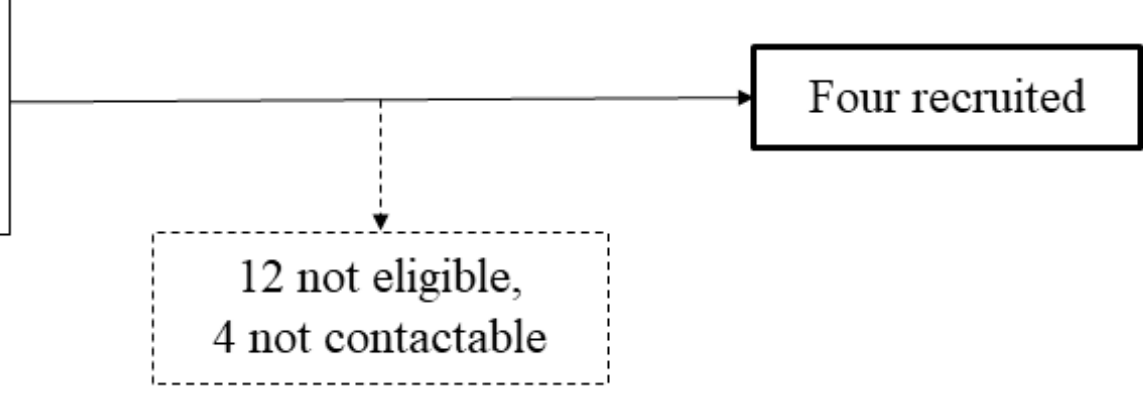

4 not contactable

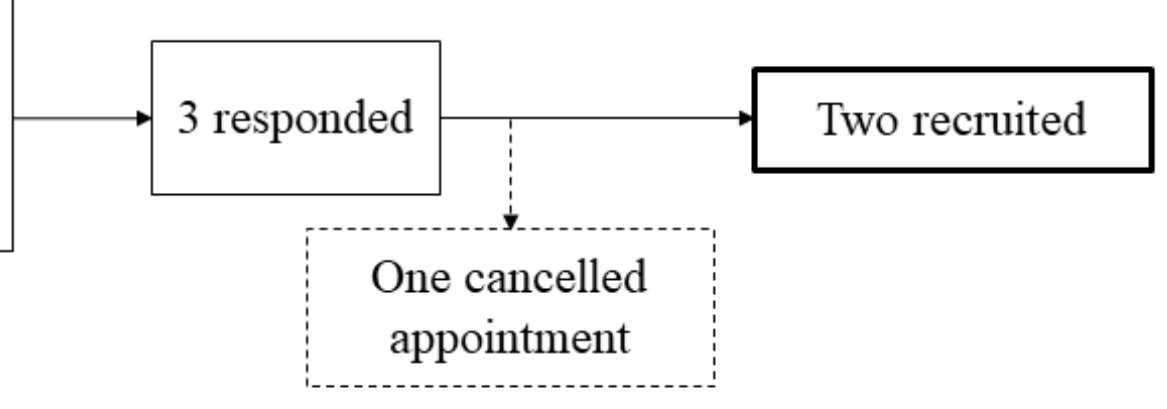


Figure 1

Recruitment flowchart. UMN: University of Minnesota. PM\&R: Physical Medicine and Rehabilitation.
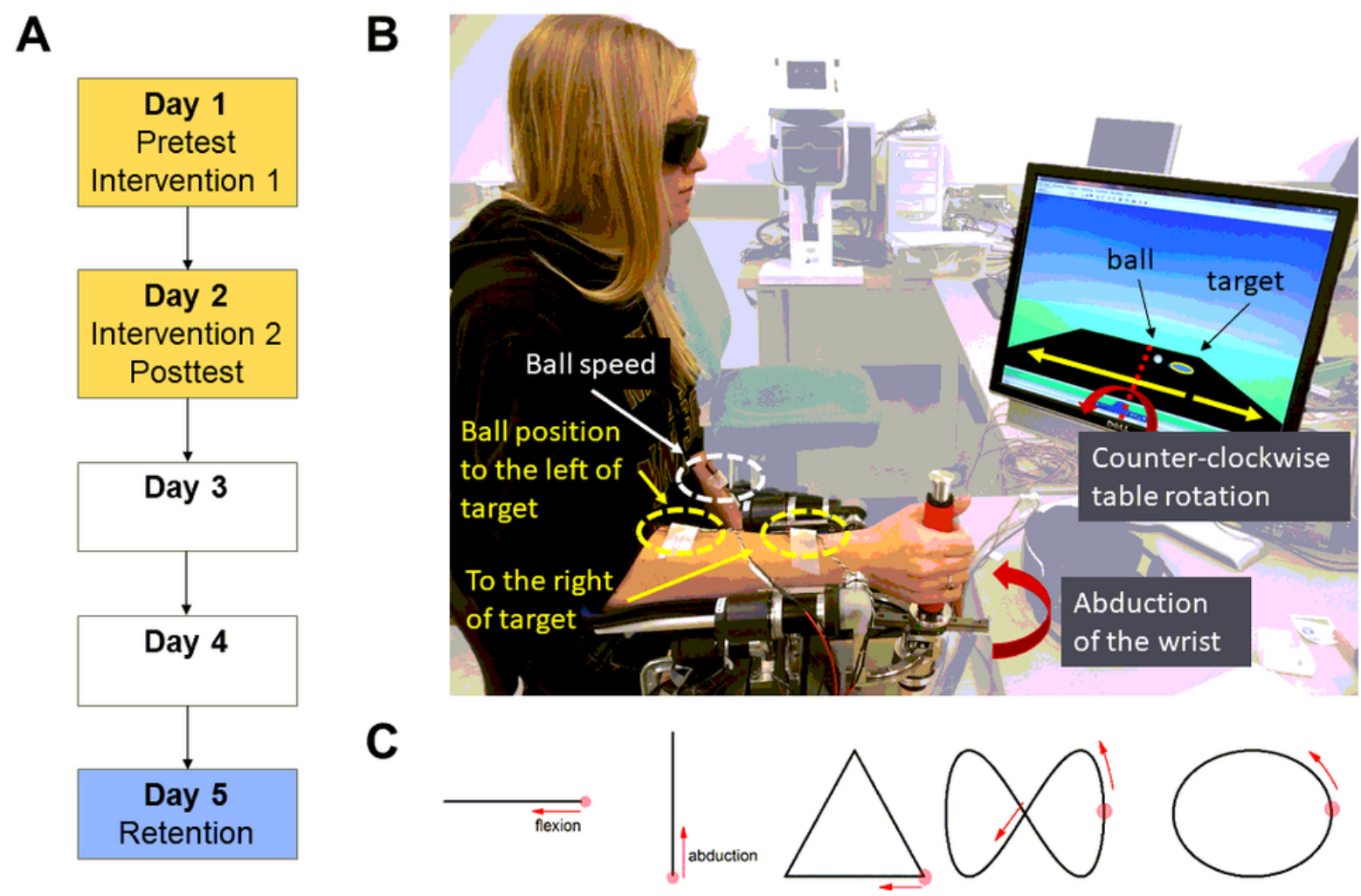

\section{Figure 2}

A. Study timeline. B. Experimental setup of the robot and the virtual ball balancing task. Wrist abduction tilted the virtual table seen in the computer display toward the left, adduction toward the right, as indicated by the maroon arrows. The task was to move the virtual ball rolled into the blue target circle. The two vibration motors attached to the skin arm indicated the respective ball position relative to the target. The distal motor turned on when the ball was on the right side of the target, the proximal motor when the ball was to the left side. Distance from the target was frequency coded (frequency increased with increasing distance to the target. The motor on the non-trained side indicated the ball speed. C. Figures of the untrained wrist tracing task. Red circles and arrows indicate the starting point and movement direction for right-handed users. 
A

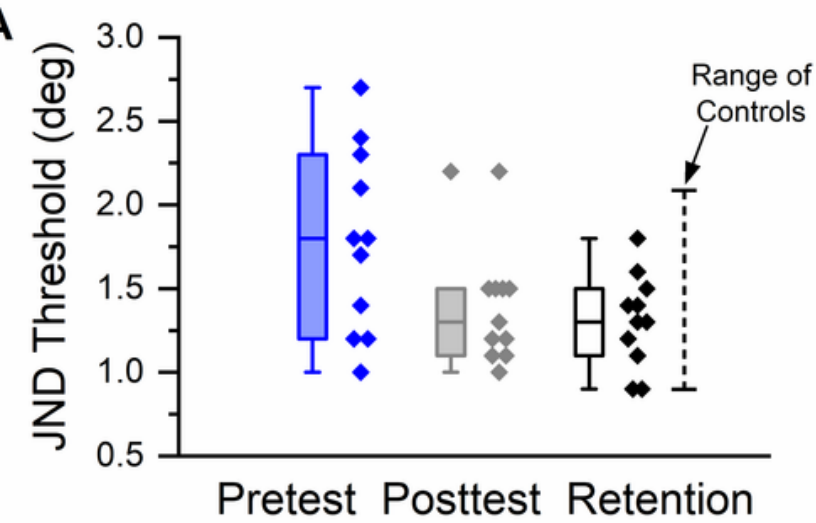

B
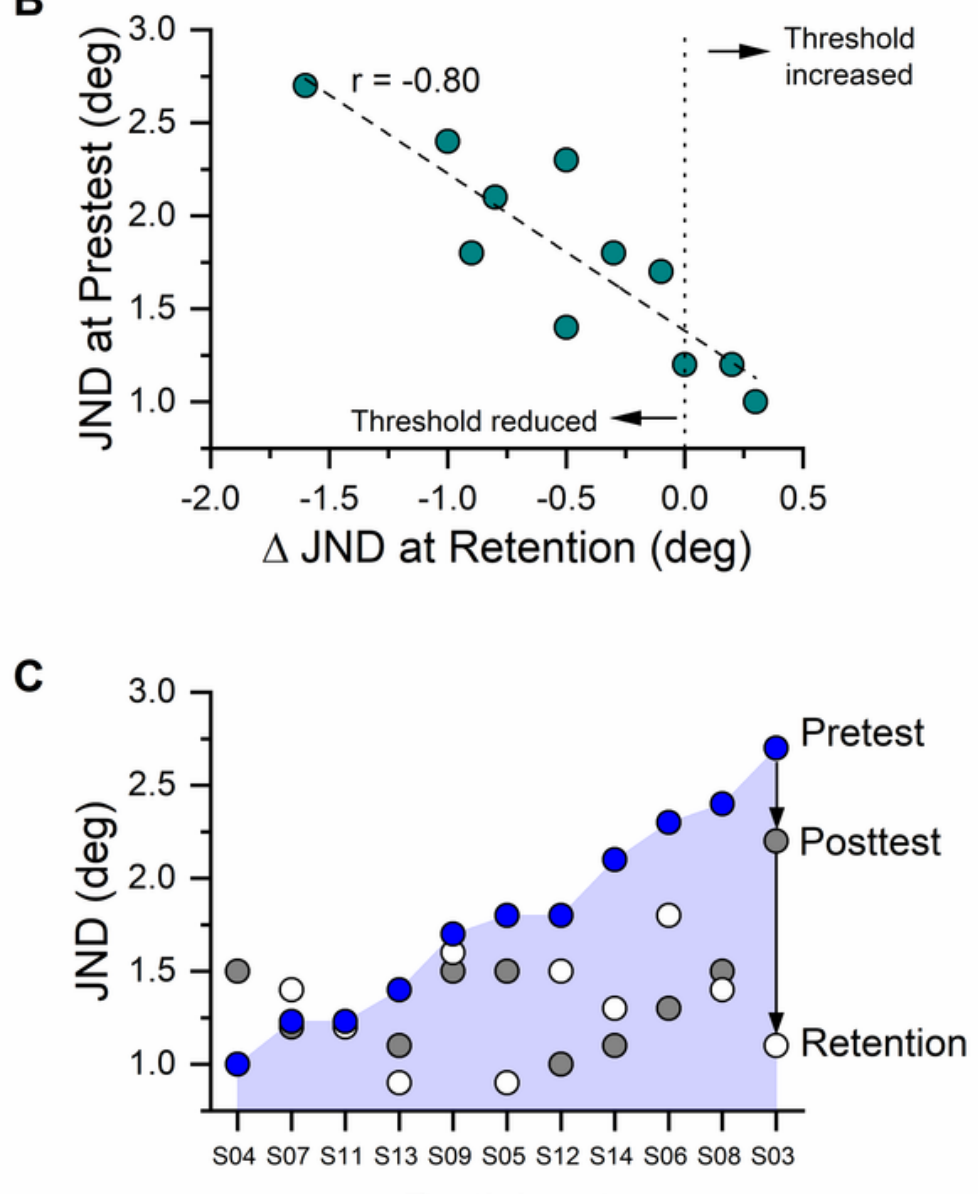

Participant

Figure 3

A. Boxplot of Just-Noticeable Difference (JND) position sense thresholds at pretest, posttest and retention. Each box indicates the interquartile range (IQR). The line within the box indicates the median. Whiskers represent the 1st and 99th percentile. Adjacent diamond symbols show all individual subject JNDs. The range of JND thresholds observed in healthy age-matched controls are indicated in the right. B. Correlation between JND thresholds at pretest in relation to change in JND at retention. A negative 
value indicates a reduction in threshold, i.e. an improvement in proprioceptive acuity. C. Change in JND for each participant as a function of training. Data are sorted in ascending order for the pretest value. Grey and white circles indicate the related values at posttest and retention.

A

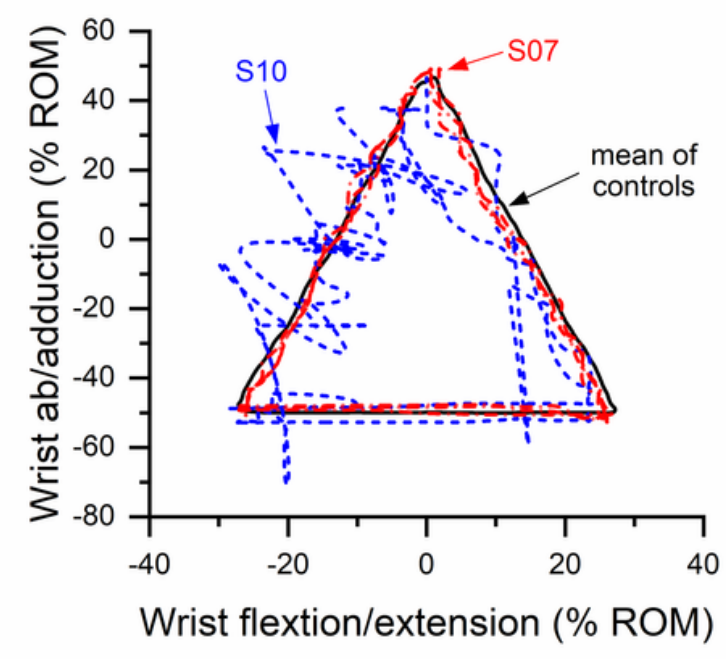

B

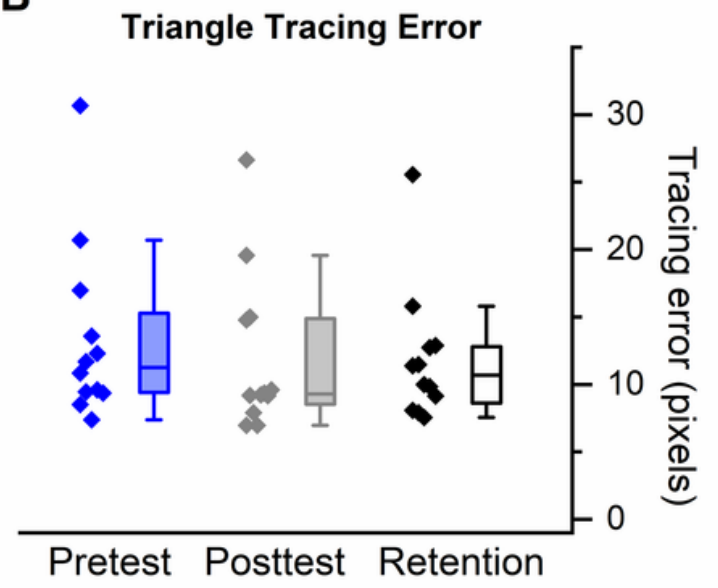

\section{Figure 4}

A. Exemplar wrist tracing performance at pretest for two stroke participants $(\mathrm{S} 07, \mathrm{~S} 10)$ during the triangle tracing task. The black triangle represents the mean trajectory of the controls. S07 demonstrated comparable performance to controls, while S10 exhibited a large tracing error. B. Boxplot of triangle tracing error at pretest, posttest and retention. Each box indicates the distribution from the 25-75th percentile. The line within the box indicates the median. Whiskers represent the 1st and 99th percentile. Adjacent diamond symbols show all individual subject mean tracing errors values. 

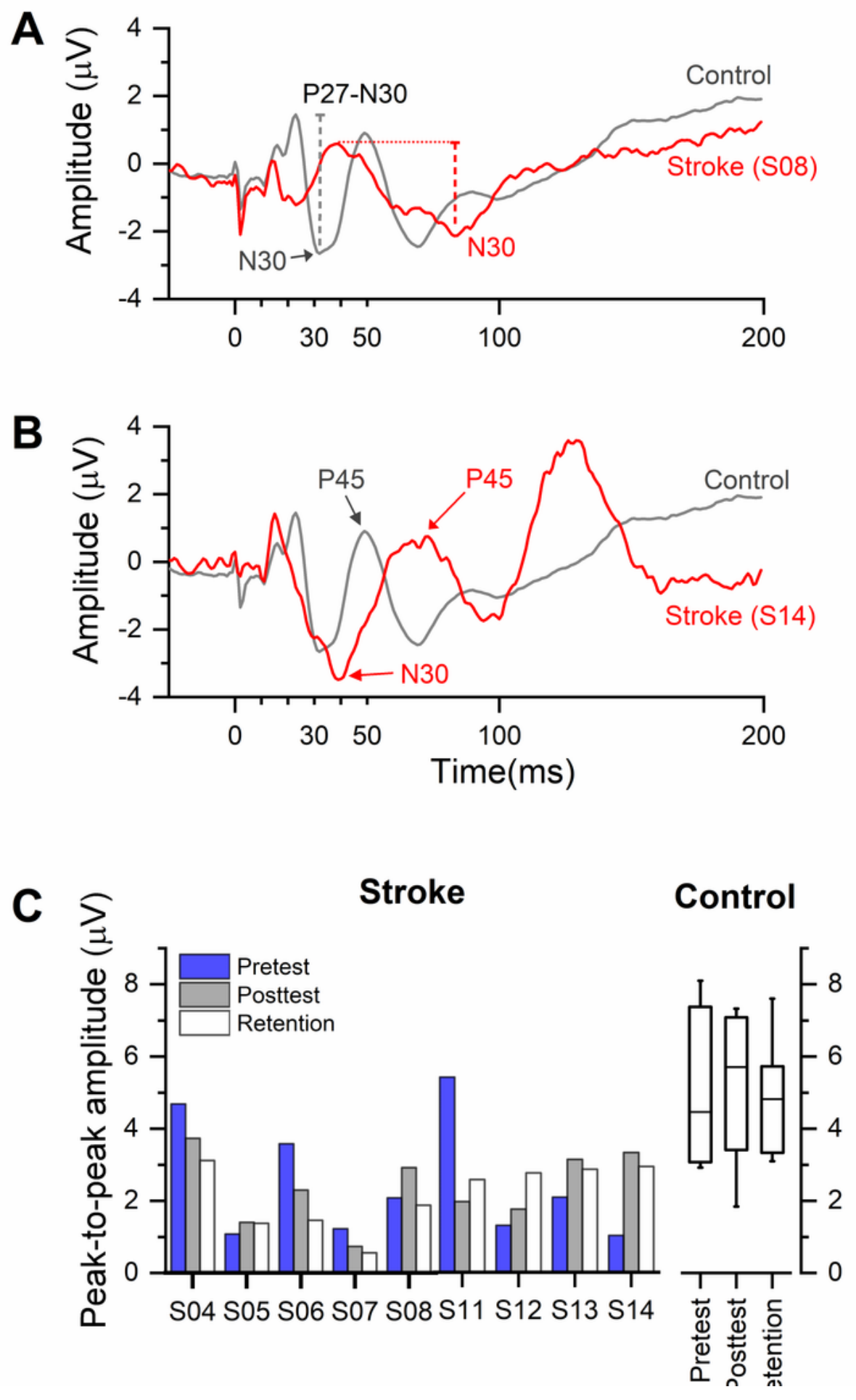

Figure 5

Median nerve SEP time-series data of two stroke participants and related summary data. A. Stroke participant S08 exhibited a longer N30 latency and a decreased P27-N30 SEP amplitude in comparison to the average waveform of the control group. B. Stroke participant S14 showed prolonged N30 and P45 latencies when compared to controls. C. P27-N30 peak-to-peak amplitude across visits. Data of individual 
stroke participants and the summary statistics of the control group were shown: medians (lines), IQR (box boundary) and 5th - 95th percentiles (whiskers). 